Известия НАН Армении, Физика, т.57, №1, с.59-69 (2022)

УДК 539.1

DOI:10.54503/0002-3035-2022-57.1-59

\title{
СПИН-ЗАВИСИМОЕ МНОГОКАНАЛЬНОЕ РАССЕЯНИЕ ЭЛЕКТРОНА В КВАНТОВОЙ ПРОВОЛОКЕ С ТОЧЕЧНЫМ ДЕФЕКТОМ
}

\author{
Д.А. БАДАЛЯН, А.Ж. МУРАДЯН* \\ Ереванский государственный университет, Ереван, Армения \\ *e-mail: muradyan@ysu.am \\ (Поступила в редакцию 18 октября 2021 г.)
}

\begin{abstract}
Рассмотрено туннельное прохождение микрочастицы со спином через трехмерный дельта-образный потенциальный барьер, вложенный в квантовую проволоку с характерным эффектом размерного квантования. Этот эффект приводит к многоканальному рассеянию, не возникающему в одномерных задачах. На частицу действует также магнитное поле, направленное вдоль проволоки и принимающее в общем случае неравные постоянные значения с обеих сторон барьера. Получены аналитические выражения для коэффициентов рассеяния и коэффициента спиновой поляризации многоканального рассеяния. Рассмотрен ряд частных случаев полученных формул. В одноканальном приближении подробно исследованы зависимости упомянутых коэффициентов от энергии частицы, параметров потенциального барьера и нанотрубки, магнитного поля.
\end{abstract}

\section{1. Введение}

Большое внимание к квантовым проволокам (квантовым нитям, нанотрубкам, одномерным проводникам и т.д.) обусловлено уникальными свойствами этих систем, которые в зависимости от характера симметрии кристаллической решетки материала могут проявлять как неметаллические, так и полупроводниковые свойства. Процессы переноса заряда тока в одномерных проводниках обладают рядом особенностей, не встречающихся в объемных веществах. Это связано со своеобразным характером межмолекулярного взаимодействия в одном измерении. Из-за низкой размерности проводника меняется также действие различных точечных дефектов (примесей, вакансий), которые влияют на ход транспорта частиц. Например, установлено, что проводимость нанотрубки сильно подавляется даже единственной примесью [1-3]. Аналогичные явления могут иметь место в области спинтроники, центральным направлением которой является исследование спин-зависимости транспорта в низкоразмерных структуpax.

В настоящей работе рассматривается задача туннельного рассеяния электрона, с учетом его спина, на нейтральном точечном дефекте, при наличии внешнего магнитного поля. Точечный дефект моделируется трехмерным $\delta$-образным 
потенциалом. Магнитное поле описывается ступенчатой функцией.

Следует сказать, что туннельным явлениям спиновых частиц в магнитных гетероструктурах посвящено значительное число теоретических работ. Особенно часто используется одномерная модель с прямоугольными потенциальными барьерами. Однако, в нанотрубке, содержащей трехмерный точечный дефект, необходимо учитывать не только продольное, но и поперечное движение электрона. Ограничение в поперечном движении, из-за эффекта размерного квантования, приводит к дискретному спектру «поперечной» энергии. Энергия частицы при рассеянии равняется сумме энергий продольного и поперечного движений. В этих условиях при упругом рассеянии в продольном направлении частица может перейти на другой уровень в поперечном движении с возникновением нового канала рассеяния.

В работе [4], без учета магнитного поля и спина, получены все амплитуды многоканального рассеяния при прохождении электрона через барьеры, состоящие из одного и двух трехмерных $\delta$-образных потенциалов. В настоящей работе исследуется характер влияния магнитного поля на рассеяние микрочастицы со спином в квантовой проволоке с трехмерным $\delta$-потенциалом.

\section{2. Основные уравнения}

Рассмотрим квантовую проволоку цилиндрической формы, разделенной на две части тонким слоем изолятора ( $\delta$-легированная примесь). Слева и справа от примеси включены статические магнитные поля $\mathbf{H}_{1}$ и $\mathbf{H}_{2}$, которые направлены вдоль оси $z$, совпадающей с осью цилиндра. Электрон с энергией $E$, двигаясь по трубке слева налетает на примесь. Потенциал примеси описывается функцией $U(x, y, z)$. Энергия взаимодействия собственного магнитного момента электрона $\boldsymbol{\mu}$ с внешним магнитным полем описывается членом

$$
U_{\mathrm{M}}=-(\boldsymbol{\mu} \cdot \mathbf{H})=\mu_{0} \sigma_{\mathrm{z}} H_{\mathrm{z}},
$$

где

$$
H_{\mathrm{z}}=\left\{\begin{array}{ll}
H_{1}, & z<z_{0} \\
H_{2}, & z>z_{0}
\end{array} .\right.
$$

$z_{0}$ является $z$-координатой примеси, $\mu_{0}$-магнетон Бора, $\sigma_{z}$ принимает значения \pm 1 , где верхний знак соответствует состоянию, в котором спин электрона направлен по оси $z$, а нижний знак - против оси $z$. Если пренебречь влиянием внешнего магнитного поля на импульс микрочастицы, что верно при относительно высоких температурах и слабых магнитных полях [5], то уравнение Паули, с учетом формулы (1), можно представить в виде двух обычных уравнений Шредингера

$$
\begin{aligned}
& \left(-\frac{\hbar^{2}}{2 m} \Delta+U(x, y, z)+\mu_{0} H_{z}-E\right) \psi^{+}(x, y, z)=0, \\
& \left(-\frac{\hbar^{2}}{2 m} \Delta+U(x, y, z)-\mu_{0} H_{z}-E\right) \psi^{-}(x, y, z)=0,
\end{aligned}
$$


где волновая функция $\psi^{+}$описывает состояние, в котором спин электрона направлен по оси $z$, а волновая функция $\psi^{-}$- против оси $z$. Переходя к цилиндрическим координатам, вместо уравнений (2) получим

$$
\left(\frac{1}{\rho} \frac{\partial}{\partial \rho}\left(\rho \frac{\partial}{\partial \rho}\right)+\frac{1}{\rho^{2}} \frac{\partial^{2}}{\partial \varphi^{2}}+\frac{\partial^{2}}{\partial z^{2}}+\chi^{2}-V(\rho, \varphi, z) \mp h(z)\right) \psi^{ \pm}(\rho, \varphi, z)=0,
$$

где $\chi^{2}=\left(2 m / \hbar^{2}\right) E, V(\rho, \varphi, z)=\left(2 m / \hbar^{2}\right) U(\rho, \varphi, z), h(z)=\mu_{0}\left(2 m / \hbar^{2}\right) H_{z}$.

Уравнение (3) удовлетворяет граничному условию

$$
\psi^{ \pm}(\rho, \varphi, z)=0 \text {, если } \rho \geq a,
$$

где $a-$ радиус кругового сечения нанотрубки.

Решение уравнения (3) с граничным условием (4) можно представить в виде разложения по ортогональным функциям $R_{n l}(\rho) \cos l \varphi$ :

$$
\psi^{ \pm}(\rho, \varphi, z)=\sum_{n=1}^{\infty} \sum_{l=0}^{\infty} \psi_{n l}^{ \pm}(z) R_{n, l}(\rho) \cos l \varphi
$$

где $R_{n l}(\rho)$ является решением уравнения

$$
\left(\frac{d^{2}}{d \rho^{2}}+\frac{1}{\rho} \frac{d}{d \rho}+\chi_{n l}^{2}-\frac{l^{2}}{\rho^{2}}\right) R_{n l}(\rho)=0
$$

и имеет вид

$$
R_{n l}(\rho)=\frac{J_{l}\left(\chi_{n l} \rho\right)}{\sqrt{\pi} a J_{l+1}\left(\chi_{n l} \rho\right)} .
$$

Здесь $J_{l}(\cdots)$ - цилиндрическая функция Бесселя первого рода и величина $\chi_{n l}$ определяется из условия (4), которое принимает вид

$$
J_{l}\left(\chi_{n l} a\right)=0 .
$$

Решения трансцендентного уравнения (8) хорошо известны: наименьшее значение $\chi_{n, l}$, соответствующее паре индексов $n=1, l=0$, определяется из равенства $\chi_{10} \cdot a=2.405$. Следующий корень с индексами $n=1, l=1$ дает $\chi_{11} \cdot a=3.832$ и т.д.

В разложении (5) функции $\psi_{n l}^{ \pm}(\rho)$ являются решениями системы связанных дифференциальных уравнений

$$
\frac{d^{2} \psi_{n l}^{ \pm}(z)}{d z^{2}}+\varepsilon_{n l}^{ \pm}(z) \psi_{n l}^{ \pm}(z)-\sum_{n^{\prime}=1}^{\infty} \sum_{l^{\prime}=0}^{\infty} V_{n l ; n^{\prime} l^{\prime}}(z) \psi_{n^{\prime} l^{\prime}}^{ \pm}(z)=0,
$$

где

$$
V_{n l ; n^{\prime} l^{\prime}}(z)=\int_{0}^{a}\left(\rho R_{n l}(\rho) R_{n^{\prime} l^{\prime}}(\rho) \int_{0}^{2 \pi} V(\rho, \varphi, z) \cos l \varphi \cos l^{\prime} \varphi d \varphi\right) d \rho
$$

и

$$
\varepsilon_{n, l}^{ \pm}(z)=\chi^{2}-\chi_{n l}^{2} \mp h(z)
$$




\section{3. Амплитуды спин-зависимого многоканального рассеяния электрона на трехмерном $\delta$-образном потенциале}

Получим амплитуды прохождения и отражения в частном случае потенциала, представляющего собой трехмерную $\delta$-функцию, т.е. потенциал нулевого радиуса взаимодействия. Пусть потенциал помещен в точке нанотрубки с радиус-вектором $\boldsymbol{r}_{0}=\left(\rho_{0} \cos \varphi_{0}, \rho_{0} \sin \varphi_{0}, 0\right)$ :

$$
V(\rho, \varphi, z)=\lambda \delta\left(\rho \cos \varphi-\rho_{0} \cos \varphi_{0}\right) \delta\left(\rho \sin \varphi-\rho_{0} \sin \varphi_{0}\right) \delta(z),
$$

где $\lambda$ - мощность $\delta$-потенциала. Подставляя формулу (12) в интеграл (10), получим

$$
V_{n l ; n^{\prime} l^{\prime}}(z)=c_{n l} c_{n^{\prime} l^{\prime}} \delta(z)
$$

где

$$
c_{n l}=\sqrt{\frac{\lambda}{\pi a^{2}}} \frac{J_{l}\left(\chi_{n l} \rho_{0}\right)}{J_{l+1}\left(\chi_{n l} a\right)},
$$

где для конкретности выбран $\varphi_{0}=0$.

Подстановка (13) в уравнение (9) дает

$$
\frac{d^{2} \psi_{n l}^{ \pm}(z)}{d z^{2}}+\varepsilon_{n l}^{ \pm}(z) \psi_{n l}^{ \pm}(z)-\left(c_{n l} \sum_{n^{\prime}=1}^{\infty} \sum_{l^{\prime}=0}^{\infty} c_{n^{\prime} l^{\prime}} \psi_{n^{\prime} l^{\prime}}^{ \pm}(z)\right) \delta(z)=0 .
$$

Уравнение (15) описывает продольное спин-зависимое движение электрона по каналу, характеризуемому индексами $(n, l)$ и знаком проекции спина. Полное число каналов рассеяния равняется числу поперечных уровней энергии частицы, умноженному на два. Условимся эти уровни нумеровать согласно росту значений поперечной энергии. Далее предположим, что число этих уровней и, следовательно, число каналов рассеяния с фиксированным направлением спина («+» или «-») конечно и равно $N$. Для нумеровки каналов рассеяния удобно вместо индексов $(n, l)$ использовать индекс $m$, который принимает значения $m=1,2, \cdots N$. В соответствии с этим, систему уравнений (13) перепишем в виде

$$
\frac{d^{2} \psi_{m}^{ \pm}(z)}{d z^{2}}+\varepsilon_{m}^{ \pm}(z) \psi_{m}^{ \pm}(z)-\delta(z)\left(\sum_{m^{\prime}=1}^{N} A_{m m^{\prime}} \psi_{m^{\prime}}^{ \pm}(z)\right)=0 .
$$

где

$$
A_{m m^{\prime}}=c_{m} c_{m^{\prime}} .
$$

В формуле (16) величина $\varepsilon_{m}^{ \pm}(z)$ содержит ступенчатую функцию $h(z)$. Поэтому, в дальнейшем, имея в виду формулы (1'), воспользуемся следующим условием:

$$
\varepsilon_{m}^{ \pm}(z)=\left\{\begin{array}{c}
\varepsilon_{m}^{ \pm}\left(h_{1}\right)=\chi^{2}-\chi_{m}^{2} \mp h_{1} \quad z<0 \\
\varepsilon_{m}^{ \pm}\left(h_{2}\right)=\chi^{2}-\chi_{m}^{2} \mp h_{2}, \quad z>0
\end{array} .\right.
$$

Приступим теперь к нахождению амплитуд рассеяния электрона. Пусть частица с произвольным волновым вектором $k_{p}^{ \pm}(z)=\sqrt{\varepsilon_{p}^{ \pm}(z)}(p<N)$ проходит 
сквозь $\delta$-образный барьер. Асимптотическими решениями системы (16), при $\varepsilon_{m}^{ \pm}(z)>0$, являются плоские волны

$$
\psi_{m}^{ \pm}(z)=\left\{\begin{array}{ll}
\delta_{m p} \exp \left(i k_{m}^{ \pm}\left(h_{1}\right) z\right)+r_{m}^{ \pm}\left(h_{1}\right) \exp \left(-i k_{m}^{ \pm}\left(h_{1}\right) z\right), & z<0 \\
t \frac{ \pm}{m}\left(h_{2}\right) \exp \left(i k_{m}^{ \pm}\left(h_{2}\right) z\right), & z>0
\end{array},\right.
$$

где $r_{m}^{ \pm}$и $r_{m}^{ \pm}$- амплитуды прохождения и отражения по $m$-му каналу. Условия сшивания волновых функций $\psi_{m}^{ \pm}(z)$ и их производных при прохождении частицы через сингулярную точку $z=0$ имеют вид

$$
\begin{gathered}
\psi_{m}^{ \pm}(+0)=\psi_{m}^{ \pm}(-0), \\
\frac{d \psi_{m}^{ \pm}(+0)}{d z}-\frac{d \psi_{m}^{ \pm}(-0)}{d z}=\sum_{m^{\prime}=1}^{N} A_{m m^{\prime}} \psi_{m^{\prime}}^{ \pm}(0) .
\end{gathered}
$$

Используя формулы (19), (20) получим

$$
\begin{gathered}
t_{m}^{ \pm}=\delta_{m p}+r_{m}^{ \pm}, \\
i\left(k_{m}^{ \pm}\left(h_{1}\right)+k_{m}^{ \pm}\left(h_{2}\right)\right) t_{m}^{ \pm}-2 i k_{m}^{ \pm}\left(h_{1}\right) \delta_{m p}=\sum_{m^{\prime}=1}^{N} A_{m m^{\prime}} t_{m^{\prime}}^{ \pm}
\end{gathered}
$$

Система уравнений (21), с учетом двух ориентаций спина, содержит $4 N$ неизвестных амплитуд рассеяния. В действительности, достаточно найти только амплитуды прохождения $t_{m}^{ \pm}$, так как из первой формулы (21) видно, что при $m=p, t_{p}^{ \pm}=1+r_{p}^{ \pm}$, а при $m \neq p, t_{m}^{ \pm}=r_{m}^{ \pm}$. Это свойство вытекает из условия непрерывности волновой функции при прохождении частицы через одиночный $\delta$ образный барьер.

Далее, второе из уравнений (21) запишем в виде

$$
\sum_{m^{\prime}=1}^{N} B_{m m^{\prime}}^{ \pm} t_{m^{\prime}}^{ \pm}=-2 i k_{m}^{ \pm}\left(h_{1}\right) \delta_{m p},
$$

где

$$
B_{m m^{\prime}}^{ \pm}=A_{m m^{\prime}}-i\left(k_{m^{\prime}}^{ \pm}\left(h_{1}\right)+k_{m^{\prime}}^{ \pm}\left(h_{2}\right)\right) \delta_{m m^{\prime}} .
$$

Из $N$ неизвестных $t_{m}^{ \pm}$выделим амплитуду прохождения $t_{p}^{ \pm}$, которая определяется следующей формулой Крамера:

$$
t_{p}^{ \pm}=D_{p}^{ \pm} / D^{ \pm}
$$

где

$$
D^{ \pm}=\left|\begin{array}{cccc}
B_{11}^{ \pm} & A_{12} & \cdots & A_{1 N} \\
A_{21} & B_{22}^{ \pm} & \cdots & A_{2 N} \\
\cdots & \cdots & \cdots & \cdots \\
A_{N 1} & A_{N 2} & \cdots & B_{N N}^{ \pm}
\end{array}\right| .
$$

Вычисление определителя (25) выполняется по формуле, полученной в [6]: 


$$
\begin{aligned}
D^{ \pm} & =\left(1+\sum_{m=1}^{N} \frac{A_{m m}}{B_{m m}^{ \pm}-A_{m m}}\right) \prod_{m^{\prime}=1}^{N}\left(B_{m}^{ \pm}-A_{m m}\right) \\
& =\left(1+\sum_{m=1}^{N} \frac{A_{m m}}{B_{m m}^{ \pm}-A_{m m}}\right) \prod_{m^{\prime}=1}^{N}\left[-i\left(k_{m^{\prime}}^{ \pm}\left(h_{1}\right)+k_{m^{\prime}}^{ \pm}\left(h_{2}\right)\right)\right] .
\end{aligned}
$$

Определитель $D_{p}^{ \pm}$получается из $D^{ \pm}$заменой его $p$-го столбца столбцом свободных членов системы (22). Используя формулу (26) получим

$$
D_{p}^{ \pm}=-2 i k_{p}^{ \pm}\left(h_{1}\right)\left(1+i \sum_{m=1}^{N} \frac{A_{m m}}{k_{m}^{ \pm}\left(h_{1}\right)+k_{m}^{ \pm}\left(h_{2}\right)}\right) \prod_{m^{\prime}=1}^{N}{ }^{\prime}\left[-i\left(k_{m^{\prime}}^{ \pm}\left(h_{1}\right)+k_{m^{\prime}}^{ \pm}\left(h_{2}\right)\right)\right],
$$

где штрих у знаков суммы и произведения означает, что при выполнении этих действий надо опустить члены с $m=p$ и $m^{\prime}=p$. Из формул (24), (26) и (27) следует, что

$$
\begin{aligned}
& t_{p}^{ \pm}=\frac{2 k_{p}^{ \pm}\left(h_{1}\right)}{k_{p}^{ \pm}\left(h_{1}\right)+k_{p}^{ \pm}\left(h_{2}\right)}\left(1-i \frac{A_{p p} G^{ \pm}\left(h_{1}, h_{2}\right)}{k_{m}^{ \pm}\left(h_{1}\right)+k_{m}^{ \pm}\left(h_{2}\right)}\right), \quad r_{p}^{ \pm}=t_{p}^{ \pm}-1, \\
& G^{ \pm}\left(h_{1}, h_{2}\right)=\left(1+i \sum_{m=1}^{N} \frac{A_{m m}}{k_{m}^{ \pm}\left(h_{1}\right)+k_{m}^{ \pm}\left(h_{2}\right)}\right)^{-1} .
\end{aligned}
$$

В частности, при рассмотрении рассеяния по первому каналу $(p=1)$ получим

$$
t_{1}^{ \pm}=\frac{2 k_{1}^{ \pm}\left(h_{1}\right)}{k_{1}^{ \pm}\left(h_{1}\right)+k_{1}^{ \pm}\left(h_{2}\right)}-i \frac{2 k_{1}^{ \pm}\left(h_{1}\right) A_{11} G^{ \pm}\left(h_{1}, h_{2}\right)}{\left(k_{1}^{ \pm}\left(h_{1}\right)+k_{1}^{ \pm}\left(h_{2}\right)\right)^{2}}, r_{1}^{ \pm}=t_{1}^{ \pm}-1,
$$

где $k_{1}^{ \pm}\left(h_{1}\right)=\sqrt{\chi^{2}-\chi_{1}^{2} \mp h_{1}}, \quad k_{2}^{ \pm}\left(h_{2}\right)=\sqrt{\chi^{2}-\chi_{2}^{2} \mp h_{2}}$. Формулы для амплитуд $t_{m \neq 1}^{ \pm}=r_{m \neq 1}^{ \pm}$получаются аналогичным образом. Эти формулы не приводятся здесь ввиду их громоздкости. Более простые выражения получаются в частном случае, когда магнитное поле является однородным $\left(h_{1}=h_{2}=h\right)$. Тогда получим

$$
t_{1}^{ \pm}=1-i \frac{A_{11}}{2 k_{1}^{ \pm}(h)} G^{ \pm}(h), t_{m \neq 1}^{ \pm}=-i \frac{A_{1 m}}{2 k_{m}^{ \pm}(h)} G^{ \pm}(h),
$$

и аналогично для коэффициентов отражения $r_{m}^{ \pm}$. Эти равенства формально совпадают с соответствующими формулами, полученными в работе [4], где $\mathbf{H}=0$. В одноканальном приближении ( $N=1$ ) из (30) получим

$$
t_{1}^{ \pm}=\left(1+i \frac{A_{11}}{2 k_{1}^{ \pm}(h)}\right)^{-1}, \quad r_{1}^{ \pm}=-i \frac{A_{11}}{2 k_{1}^{ \pm}(h)}\left(1+i \frac{A_{11}}{2 k_{1}^{ \pm}(h)}\right)^{-1} .
$$

В отсутствии магнитного поля последние равенства сходятся с формулами, полученными в задаче о рассеянии бесспиновых частиц на одномерном $\delta$-образном потенциальном барьере [7]. В случае однородного магнитного поля $\left(h_{1}=h_{2}=h\right)$, используя формулы (30) можно показать, что 


$$
\sum_{m=1}^{N} \frac{k_{m}^{ \pm}(h)}{k_{1}^{ \pm}(h)}\left(\left|t_{m}^{ \pm}\right|^{2}+\left|r_{m}^{ \pm}\right|^{2}\right)=1 .
$$

Уравнение (32) выражает закон сохранения числа частиц со спином «+» и «-». В случае $h_{1} \neq h_{2}$ уравнение (32) принимает более сложный вид. Приведем соответствующую формулу только для одноканального рассеяния, которая получается из формул (29):

$$
T_{1}^{ \pm}+R_{1}^{ \pm}=1
$$

где

$$
T_{1}^{ \pm}=\frac{k_{1}^{ \pm}\left(h_{2}\right)}{k_{1}^{ \pm}\left(h_{1}\right)}\left|t_{1}^{ \pm}\right|^{2}
$$

— коэффициент прохождения или прозрачности,

$$
R_{1}^{ \pm}=\left|r_{1}^{ \pm}\right|^{2}
$$

— коэффициент отражения.

Следует отдельно заметить, что в общем случае неравных магнитных полей слева и справа начало координат коэффициент прохождения меньше единицы даже в отсутствии $\delta$-потенциального барьера. Отсюда также следует, что увеличение одного из этих факторов: разности магнитных полей и мощности $\delta-$ барьера, уменьшает значимость другого для коэффициента прохождения. Одинаковое по обе стороны барьера магнитное поле влияет на значение коэффициента прохождения частицы, уменьшая его для плюсовой компоненты спина и увеличивая его для минусовой компоненты. Это является следствием разнознакового сдвига энергетического спектра частицы для этих компонентов.

\section{4. Коэффициент спиновой поляризации при многоканальном рассеянии}

Для характеристики степени поляризации спина электрона при многоканальном рассеянии в однородном магнитном поле, наподобие одномерной задачи [8], можно ввести коэффициент спиновой поляризации $P_{N}$

$$
P_{N}=\sum_{m=1}^{N}\left(\frac{k_{m}^{+}(h)}{k_{1}^{+}(h)}\left|t_{m}^{+}\right|^{2}-\frac{k_{m}^{-}(h)}{k_{1}^{-}(h)}\left|t_{m}^{-}\right|^{2}\right) / \sum_{m=1}^{N}\left(\frac{k_{m}^{+}(h)}{k_{1}^{+}(h)}\left|t_{m}^{+}\right|^{2}+\frac{k_{m}^{-}(h)}{k_{1}^{-}(h)}\left|t_{m}^{-}\right|^{2}\right) .
$$

Из этой формулы следует, что если коэффициент прохождения электрона со спином «+» больше соответствующего коэффициента для частицы со спином «-», то $P_{N}>0$. В обратном случае $P_{N}<0$. Если же эти коэффициенты равны, то электрон не поляризуется: $P_{N}=0$.

При неоднородном магнитном поле, в общем случае, необходимо использовать более сложное определение коэффициента спиновой поляризации. Далее мы ограничимся одноканальным приближением. В этом случае 


$$
P_{1}=\frac{T_{1}^{+}-T_{1}^{-}}{T_{1}^{+}+T_{1}^{-}}
$$

Для дальнейших расчетов по формуле (37) удобно вместо коэффициентов прохождения $T_{1}^{ \pm}$ввести обратные им функции

$$
\left|Z_{1}^{ \pm}\right|^{2}=\left(T_{1}^{ \pm}\right)^{-1}=\frac{k_{1}^{ \pm}\left(h_{1}\right)}{k_{1}^{ \pm}\left(h_{2}\right)}\left|t_{1}^{ \pm}\right|^{-2} .
$$

Тогда, используя первую из формул (29), получим

$$
\left|Z_{1}^{ \pm}\right|^{2}=\frac{k_{1}^{ \pm}\left(h_{1}\right)}{k_{1}^{ \pm}\left(h_{2}\right)}\left[\left(\alpha_{1}^{ \pm}\right)^{2}+\left(\beta_{1}^{ \pm}\right)^{2}\right],
$$

где

$$
\alpha_{1}^{ \pm}=\frac{1}{2}+\frac{k_{1}^{ \pm}\left(h_{2}\right)}{2 k_{1}^{ \pm}\left(h_{1}\right)}, \quad \beta_{1}^{ \pm}=\frac{A_{11}}{2 k_{1}^{ \pm}\left(h_{1}\right)} .
$$

Из определения (38) также следует, что если коэффициенты $T_{1}^{ \pm}$ограничены интервалом [0,1], то функции $\left|Z_{1}^{ \pm}\right|^{2}$ изменяются в пределах $[1, \infty]$. Теперь, из равенств (37)-(40) получим следующее выражение:

$$
P_{1}=\frac{Q^{+}-Q^{-}}{Q^{+}+Q^{-}}
$$

где

$$
Q^{ \pm}=\frac{4 k_{1}^{ \pm}\left(h_{1}\right) k_{1}^{ \pm}\left(h_{2}\right)}{\left(k_{1}^{ \pm}\left(h_{1}\right)\right)^{2}+\left(k_{1}^{ \pm}\left(h_{2}\right)\right)^{2}+A_{11}^{2}} .
$$

Перейдем к рассмотрению частных и предельных случаев.

1. Магнитное поле отсутствует: $h_{1}=h_{2}=0$. Тогда $k_{1}^{+}(0)=k_{1}^{-}(0)$ и $P_{1}=0$.

2. Магнитное поле однородное: $h_{1}=h_{2}=h$. Из определения непосредственно следует, что $k_{1}^{ \pm}(h)=\sqrt{\chi^{2}-\chi_{1}^{2} \mp h}$. Из равенства (41) получим

$$
P_{1}=-\frac{A_{11}^{2} h}{A_{11}^{2}\left(k_{1}(0)\right)^{2}+4\left(\left(k_{1}(0)\right)^{2}-h^{2}\right)},
$$

где $k_{1}(0)=\sqrt{\chi^{2}-\chi_{1}^{2}}$. Знаменатель дроби (42), из-за $\chi^{2}-\chi_{1}^{2}>h$ положителен. Следовательно, $P_{1}<0$. Обратим внимание также на то, что если $h \rightarrow \chi^{2}-\chi_{1}^{2}$, то $P_{1}$ стремится к -1 , т.е. возникает почти полная спиновая поляризация.

3. $h_{1}=h, h_{2}=0$. Этот случай напоминает ситуацию, когда слева от потенциального барьера находится ферромагнетик, а справа - парамагнетик. Формула (41) в этом случае принимает следующий вид: 


$$
P_{1}=\frac{\gamma^{+}(h) k_{1}^{+}(h)-\gamma^{-}(h) k_{1}^{-}(h)}{\gamma^{+}(h) k_{1}^{+}(h)+\gamma^{-}(h) k_{1}^{-}(h)+k_{1}(0) k_{1}^{+}(h) k_{1}^{-}(h)},
$$

где $\gamma^{ \pm}(h)=A_{11}^{2}+2\left(k_{1}(0)\right)^{2} \pm h$. Заметим, что в данном случае, аналогично предыдущему, в пределе $h \rightarrow \chi^{2}-\chi_{1}^{2}$ волновой вектор $k_{1}^{+}(h)$ стремится к нулю, а $P_{1}$ к -1 .

4. Рассмотрим общий случай $h_{1} \neq h_{2}$, описываемой формулой (41). Обсудим только предельные случаи почти непроницаемого ( $\left.A_{11} \rightarrow \infty\right)$ и почти прозрачного ( $A_{11} \rightarrow 0$ ) барьера. Если $A_{11} \approx \infty$, то из формулы (41) получим

$$
P_{1}=\frac{k_{1}^{+}\left(h_{1}\right) k_{1}^{+}\left(h_{2}\right)-k_{1}^{-}\left(h_{1}\right) k_{1}^{-}\left(h_{2}\right)}{k_{1}^{+}\left(h_{1}\right) k_{1}^{+}\left(h_{2}\right)+k_{1}^{-}\left(h_{1}\right) k_{1}^{-}\left(h_{2}\right)} .
$$

Прежде всего отметим, что в рассматриваемом случае, согласно формулам (34), (38)-(40), $\lim _{A 1 \rightarrow \infty} T_{1}^{ \pm}=0$. То есть имеет место почти полное отражение поляризованных частиц. Степень их поляризации в слабых магнитных полях, удовлетворяющих условиям $h_{i} \ll \chi^{2}-\chi_{i}^{2}(i=1,2)$ можно оценить с помощью формул (44), (41'). Получим

$$
P_{1} \approx-\frac{h_{1}+h_{2}}{2\left(\chi^{2}-\chi_{1}^{2}\right)} .
$$

В другом крайнем случае, когда значения параметров $h_{1}, h_{2}$ близки к величине $\chi^{2}-\chi_{1}^{2}$, из формулы (44) следует, что $P_{1} \approx-1$.

Рассмотрим теперь случай почти прозрачного $\delta$-потенциального барьера $\left(A_{11} \approx 0\right)$. Использование формул (34), (38)-(40) приводит к выражению

$$
T_{1}^{ \pm}=4 \frac{k_{1}^{ \pm}\left(h_{2}\right)}{k_{1}^{ \pm}\left(h_{1}\right)}\left(1+\frac{k_{1}^{ \pm}\left(h_{2}\right)}{k_{1}^{ \pm}\left(h_{1}\right)}\right)^{-2} .
$$

Используя формулы (46), (41') можно убедится, что $T_{1}^{ \pm}$, а значит и $P_{1}$, инвариантны относительно замены $h_{1} \leftrightarrow h_{2}$. Как уже было отмечено, в обоих случаях коэффициенты прохождения $T_{1}^{+}$и $T_{1}^{-}$меньше единицы (и равняется единице только при $h_{1}=h_{2}$ ). Из этого следует, что коэффициенты отражения $R_{1}^{ \pm}$отличны от нуля, т.е. в данном случае имеет место явление надбарьерного отражения спиновых частиц.

Численные расчеты, выполнение по общим формулам (38)-(40), (41), (41'), показывают ожидаемые результаты для коэффициентов прохождения обеих компонент спина: (неодинокое) монотонное увеличение в зависимости от энергии частицы, от напряженности магнитного поля, радиуса нанотрубки, меры смещения дефекта от центра к стенкам нанотрубки и увеличение в зависимости от мощности $\delta$-потенциального барьера.

Что касается представляющего основной интерес в настоящей работе коэффициента спиновой поляризации, то он в пределе малых энергий падения равен -1 , т.е. частица после туннелирования оказывается полностью поляризованной (при этом точечный дефект предполагается на оси нанотрубки). Такое поведение 
можно объяснить тем, что при очень низких энергиях падения один из поляризаций вовсе не проходит через потенциальных барьер (полностью отражается от него). Коэффициент пропускания другой поляризации довольно близок к единице, что может быть использовано для создания хорошего поляризационного фильтра медленных частиц. С увеличением энергии частицы коэффициент поляризации уменьшается по модулю и в асимптотике приближается к нулю, т.е. к исходному неполяризованному состоянию. Это поскольку с увеличением энергии частицы коэффициенты прохождения приближаются друг к другу. Примечательно, что скорость уменьшения поляризации здесь уменьшается с увеличением напряженности магнитного поля и тем самым улучшает качество предлагаемого поляризатора. Зависимость от радиуса трубки в одномодовом приближении не представляет самостоятельного интереса, поскольку она определяет единственную энергию поперечного квантования и, следовательно, определенное преобразование энергетической зависимости. Наконец, о зависимости от смещения точечного дефекта от оси нанотрубки. Степень поляризации максимальна и в случае не столь высоких энергий близка к единице, если дефект расположен на оси нанотрубки, и s-образно переходит в нулевое исходное состояние с приближением к ее стенкам.

\section{5. Заключение}

Исследовано квантовое рассеяние электрона на точечном дефекте квантовой нанотрубки с учетом взаимодействия спина электрона с присутствующим продольным магнитным полем. Выведены аналитические формулы для всех коэффициентов многоканального прохождения и многоканального отражения электрона. Подробно рассматривается одноканальное рассеяние, представляющее основной интерес в области низких энергий рассеянного электрона, включая численные расчеты. Показано, что представленная схема может служить высококачественным селектором электронов определенной поляризации из изначально неполяризованного электронного пучка.

\section{ЛИТЕРАТУРА}

1. C.L. Kane, M.P.A. Fisher. Phys. Rev. Lett., 68, 1220 (1992).

2. K.A. Matveev, L.I. Glazman. Phys. Rev. Lett., 70, 990 (1993).

3. A. Furusaki, N. Nagaosa. Phys. Rev. B, 47, 4631 (1993).

4. Д.М. Седракян, Д.А. Бадалян, А.Ю. Алексанян. Изв. НАН Армении, Физика, 51, 452 (2016).

5. А.А. Соколов, И.М. Тернов, В.Ч. Жуковский. Квантовая Механика. Москва, Наука, 1979.

6. Д.М. Седракян, Д.А. Бадалян, Л.Р. Седракян. Изв. НАН Армении, Физика, 50, 176 (2015).

7. З. Флюгге. Задачи по квантовой механике, Том 1, Москва: Мир, 1974.

8. V.I. Perel, S.A. Tarasenko, I.N. Yassievich, et al. Phys. Rev. B, 67, 201304 (2003). 


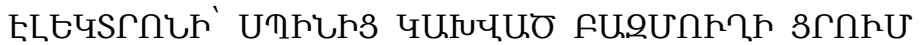

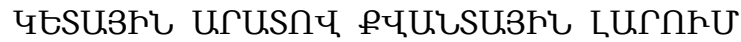

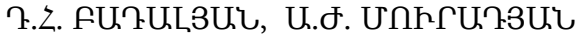

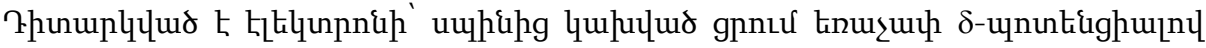

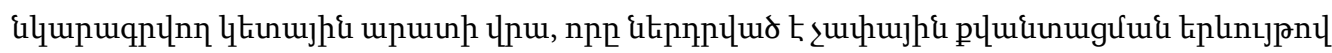

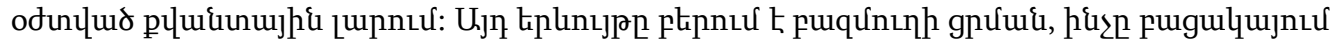

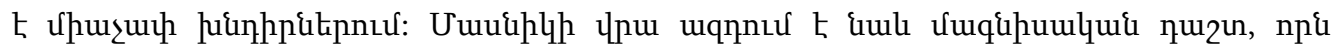

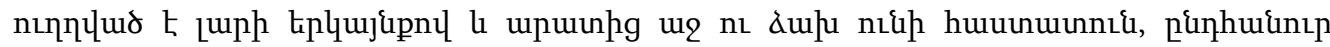

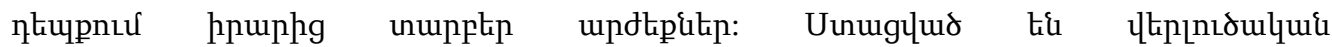

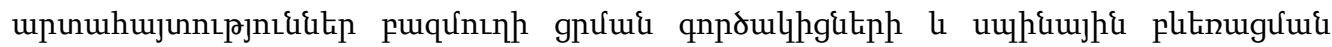

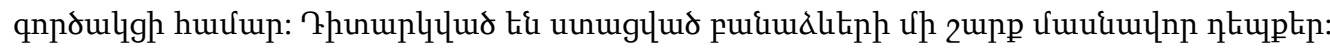

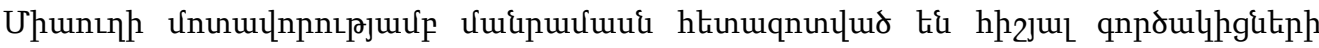

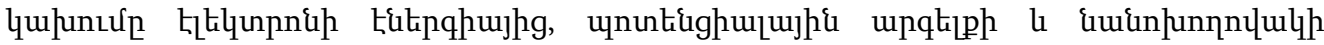

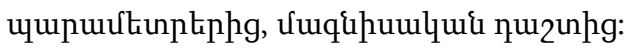

\section{SPIN-DEPENDENT MULTICHANNEL SCATTERING OF ELECTRON IN A QUANTUM WIRE WITH A POINT DEFECT}

\section{D.A. BADALYAN, A.Zh. MURADYAN}

The tunneling passage of a microparticle with spin through a three-dimensional $\delta$ shaped potential barrier embedded in a quantum wire with a characteristic effect of dimensional quantization is considered. This effect leads to multichannel scattering, which does not occur in one-dimensional problems. The particle is also affected by a magnetic field directed along the wire and generally taking unequal constant values on both sides of the barrier. Analytical expressions for the scattering coefficients and the spin polarization coefficient of multichannel scattering are obtained. A number of special cases of the obtained formulas are considered. The dependences of the mentioned coefficients on the particle energy, the parameters of the potential barrier and nanotube, and the magnetic field are investigated in detail in a single-channel approximation. 